\title{
$\underline{\mathbf{P}-55}$
}

\section{Chemical Constituents from Fruit of Commersonia Batramia}

\author{
Nik Khairunissa ${ }^{1}$, Nik Abdullah Zawawi ${ }^{*}$, Norizan Ahmat ${ }^{1}$, Zetty Shafiqa Othman ${ }^{1}$, Nurunajah Ab. \\ Ghani $^{2}$ \\ ${ }^{1}$ Faculty of Applied Sciences, UiTM Shah Alam, 40450, Shah Alam, Selangor, Faculty of Pharmacy, Puncak UiTM \\ Puncak Alam Campus, 42300 Bandar Alam, Selangor; ${ }^{2}$ Atta-ur-Rahman Institute for Natural Product Discovery, Level \\ 9, FF3 Puncak UiTM Puncak Alam Campus, 42300 Bandar Alam, Selangor; E-mail: chemtitude@yahoo.com
}

Commersonia is a genus of trees and shrubs that belongs to the subfamily of Sterculiaceae and family Malvaceae comprises about 14 species which all are endemic plants of Australia, whereas the other two occur also in Southeast Asia and the western Pacific Islands. Commersonia batramia is the solely species found in Malaysia and previous study showed that this species had been traditionally used for colds and cough, to treat rheumatism, kidney troubles and dysentery in Fuji, Japan. The fruit of Commersonia batramia collected from Machang, Kelantan was dried, ground (1 kg) and macerated in Methanol. Our present study on the fruit of C.batramia afforded two coumarins; scoparone (1), 6, 8-dimethoxycoumarin (2) and three flavonoids; kaempferol (3), chalcone (4), apigenin 5-O-glucoside (5). Previous study on the leaves of this species reported the presence of three flavonols. Structure elucidations of the compounds were accomplished by using 1D and 2D NMR, MS, IR and UV-Vis spectroscopy.

Keywords: Malvaceae, Sterculiaceae, Commersonia batramia, coumarin; flavonoid. 